\title{
A HIGH-GRADIENT CW RF PHOTO-CATHODE ELECTRON GUN FOR HIGH CURRENT INJECTORS*
}

\author{
R.A. Rimmer, JLab, Newport News, VA 23606, USA
}

\begin{abstract}
The paper describes the analysis and preliminary design of a high-gradient photo-cathode RF gun optimized for high current $\mathrm{CW}$ operation. The gun cell shape is optimized to provide maximum acceleration for the newly emitted beam while minimizing wall losses in the structure. The design is intended for use in future high-current high-power CW FELs but the shape optimization for low wall losses may be advantageous for other applications such as XFELs or Linear Colliders using high peak power low duty factor guns where pulse heating is a limitation. The concept allows for DC bias on the photocathode in order to repel ions and improve cathode lifetime.
\end{abstract}

\section{INTRODUCTION}

Re-entrant cavities have long been known to offer a significant advantage in efficiency over the traditional "pillbox" shape. This is very important for CW RF guns, as these are most often limited by wall power dissipation rather than peak surface electric field. Another major concern for high current guns is cathode lifetime, which is thought to be limited by back-bombardment by ions. This design allows for a strong DC reverse bias on the cathode to repel ions. Lastly axial symmetry is desirable to minimize deflections and distortion of the bunch. This concept uses the same coaxial cathode assembly to do double duty as the RF coupler, connected to a high-power waveguide feed.

\section{SHAPE OPTIMIZATION}

The best shape for an RF gun cavity is a complex balance between many factors including peak electric field on the cathode, launch phase, exit energy, wakefields from irises, wall losses etc. Most of these factors are improved by choosing a re-entrant shape over a traditional pillbox profile [1]. For a given stress level or surface power density a re-entrant shape allows a higher electric field on the cathode compared to pillbox-like designs [2]. Figure 1 shows one such profile that, though by no means optimized, should allow a peak surface field on the cathode of $\sim 20 \mathrm{MV} / \mathrm{m}$. This is obtained by careful optimization of the RF shape and attention to cooling and stress management in the body. Table 1 lists some of the parameters of this design, which has a similar profile to the original design for the LUX $1300 \mathrm{MHz}$ gun [3], but with a demountable cathode assembly that is electrically isolated from the cavity body. Note that the field is strongly peaked on the cathode surface, figure 2 .

\footnotetext{
*This manuscript has been authored by SURA, Inc. under Contract No. DE-AC05-84ER-40150 with the U.S. Department of Energy
}

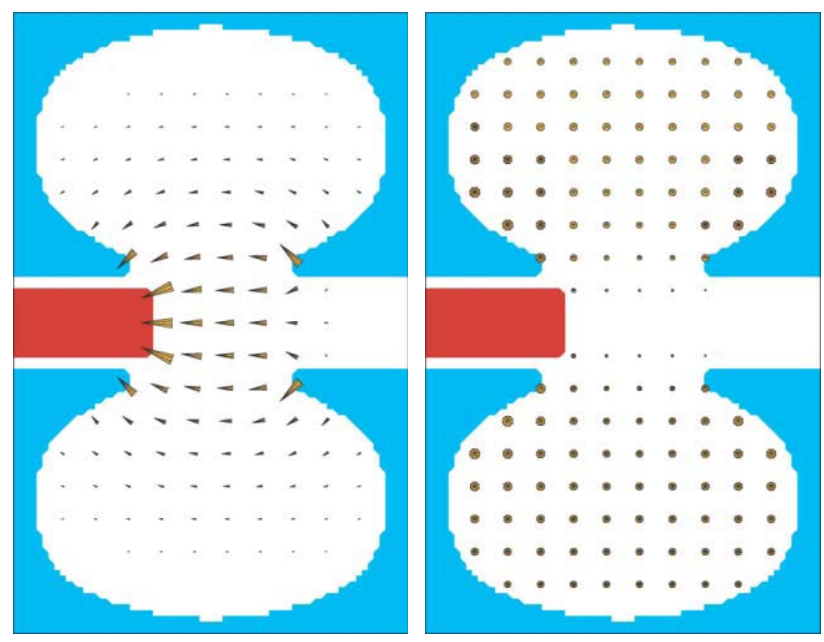

Figure 1: Electric and magnetic fields of re-entrant gun.

Table 1: RF gun parameters

\begin{tabular}{|l|c|}
\hline Frequency & $750 \mathrm{MHz}$ \\
\hline Exit energy & $\sim 1 \mathrm{MV}$ \\
\hline Peak RF field on cath. & $20 \mathrm{MV} / \mathrm{m}$ \\
\hline Field enhancement $\left(\mathrm{E}_{\max } / \mathrm{E}_{\text {cath }}\right)$ & 1.37 \\
\hline Beam power & $\leq 1 \mathrm{MW}$ \\
\hline Cavity power & $97 \mathrm{~kW}$ \\
\hline Max. wall power density & $\sim 109 \mathrm{~W} / \mathrm{cm}^{2}$ \\
\hline
\end{tabular}

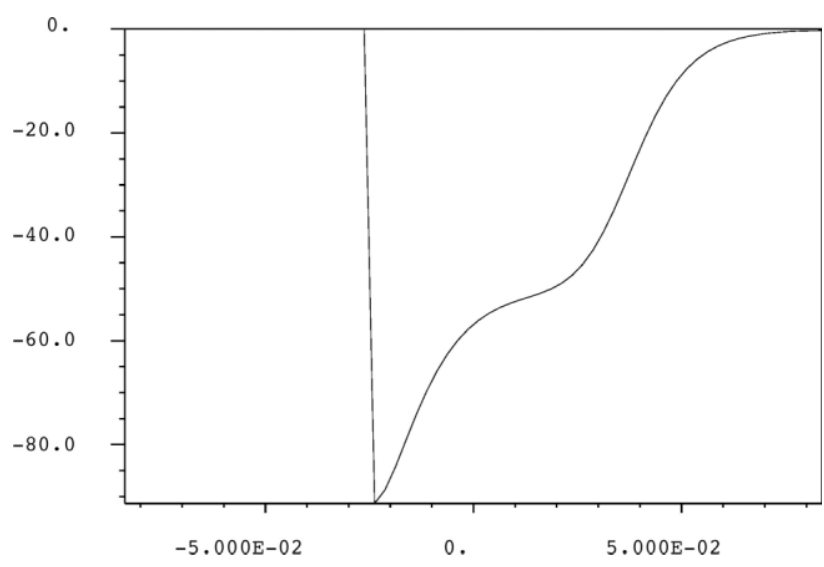

Figure 2. RF Electric field profile on axis (arbitrary units) vs length (m). 0 is the center of the cavity, cathode surface is at $-25 \mathrm{~mm}$.

\section{Wall power and stresses}

Surface power density often sets the limit to the maximum CW gradient achievable. (This is also true in the case of very high peak power pulsed guns where the limit is pulse heating). Peak surface electric field at just over 1 Kilpatrick $(\sim 25 \mathrm{MV} / \mathrm{m}$ at $750 \mathrm{MHz})$, is not expected to be a problem. Figure 3 shows contours of magnetic field strength in the gun cavity. The maxima are 
on the nosecones near the blend with the cavity wall. Note that high fields are also possible inside the cathode joint.

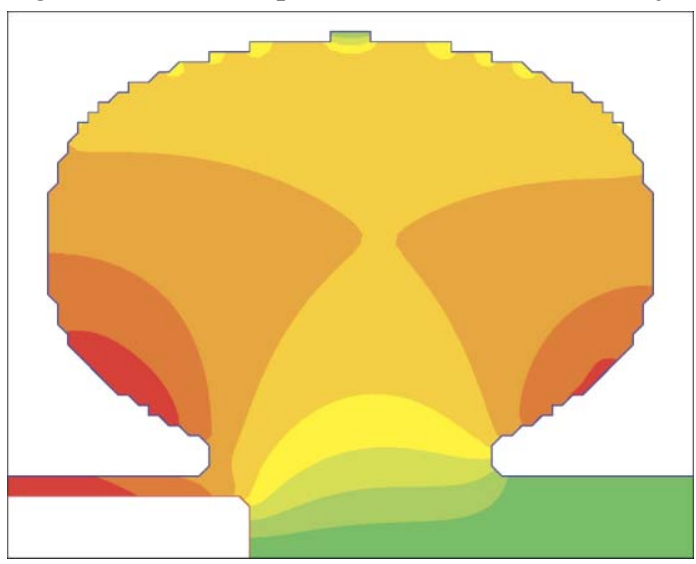

Figure 3. Contours of RF magnetic field.

Figure 4 shows an ANSYS stress analysis of the final proposed LUX gun [4,5]. The target for LUX was 64 $\mathrm{MV} / \mathrm{m}$ at $5 \%$ duty factor at $1300 \mathrm{MHz}$, with an average power of $31 \mathrm{~kW}$ and a maximum wall-power density of about $100 \mathrm{~W} / \mathrm{cm}^{2}$. This equates to about $15.5 \mathrm{MV} / \mathrm{m} \mathrm{CW}$ at $1300 \mathrm{MHz}$, with a peak surface temperature rise of $67^{\circ} \mathrm{C}$ and a maximum stress of $65 \mathrm{MPa}(\sim 9400 \mathrm{psi})$. This only about half the endurance limit of copper of $124 \mathrm{MPa}$ $(\sim 18,000 \mathrm{psi})$ for 10,000 cycles [6], (e.g. three full temperature cycles a day for $\sim 10$ years. For comparison the $476 \mathrm{MHz}$ PEP-II copper cavities were designed for a peak stress of $75 \mathrm{MPa}$ at $150 \mathrm{~kW}$ [7], and operate routinely at $\sim 100 \mathrm{~kW}$, and $50 \mathrm{MPa}(\sim 7300 \mathrm{psi})$. In fact pushing the LUX cavity to $20 \mathrm{MV} / \mathrm{m}$ only raises the stress to $108 \mathrm{MPa}$. With careful mechanical design and a few tricks in stress management (such as varying the cooling channel density and routing the water to minimize thermal gradients), these stresses could be lowered further still. Going to lower frequency also helps. For this 750 $\mathrm{MHz}$ design the maximum wall-power density is about $109 \mathrm{~W} / \mathrm{cm}^{2}$ at $20 \mathrm{MV} / \mathrm{m}$. Conventional construction techniques such as $\mathrm{NC}$ machining, e-beam welding, brazing and electroforming are adequate for these levels.

\section{Vacuum and pumping}

Good vacuum is important in any RF structure but particularly so in photocathode guns. This design is intended to be pumped through the beam iris and cathode structure, and to use a novel technique to protect the cathode from ion back-bombardment. However the LUX study has shown that if this is still insufficient additional pumping ports can be added (symmetrically), without raising the peak stresses.

\section{Demountable cathode}

The demountable cathode structure with DC isolation has many practical advantages such as rapid changing of cathodes, repair of surface damage, etc., but more importantly it allows two novel features, namely DC reverse bias to repel ions and axial RF coupling for symmetry.

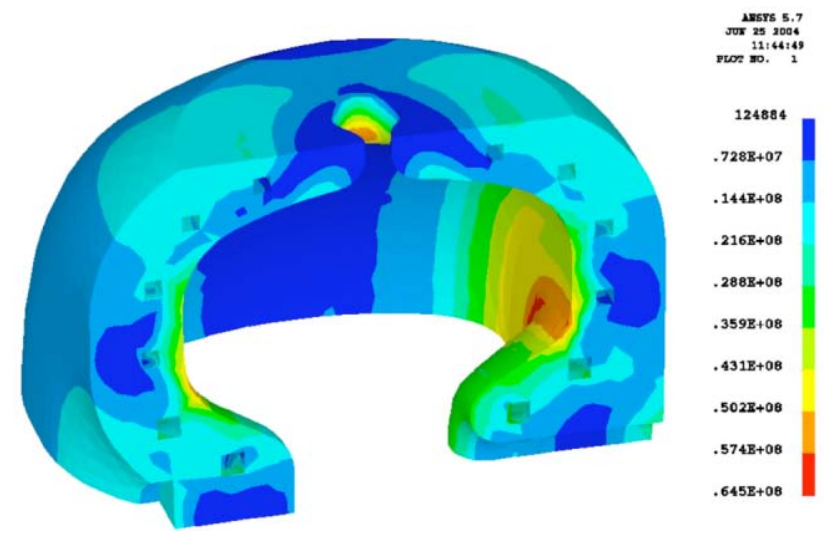

Figure 4. Stress plot for the 1.3 GHz LUX gun $\left(\mathrm{N} / \mathrm{m}^{2}\right)$.

\section{BIAS FOR ION CLEARING}

Studies have shown that ions generated by the beam can back-bombard the cathode with energies in the $\mathrm{kV}$ range [8]. With the DC isolated cathode in this design it is possible to apply a strong reverse bias voltage to repel these ions. Figure 5 shows a contour plot of the electric potential with $\mathrm{a}+10 \mathrm{kV}$ bias on the cathode. Figure 6 shows the profile on axis.

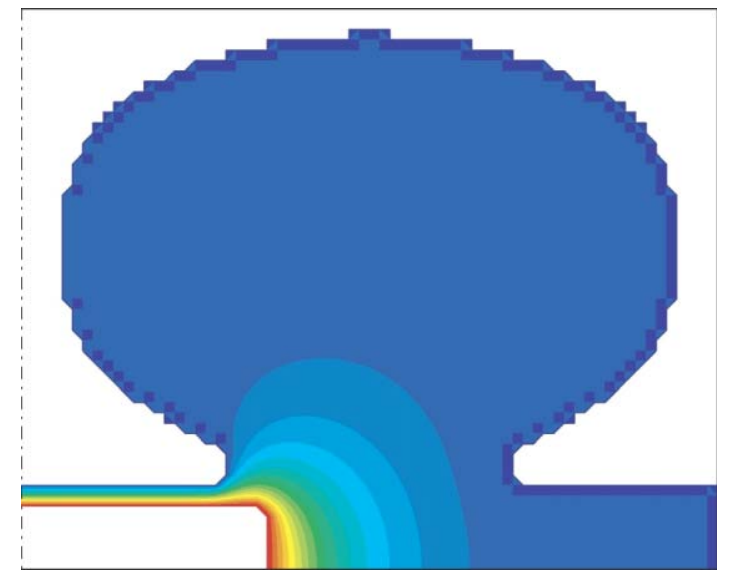

Figure 5. Contours of constant electric potential with DC bias on the cathode for ion clearing.

This bias should be strong enough to repel most ions while the DC electric field is on the cathode is less than 1 $\mathrm{MV} / \mathrm{m}$ and therefore does not significantly affect the acceleration of electrons on crest. Combined with the solenoidal magnetic field used for emittance compensation this arrangement should guide positive ions safely away from the cathode.

\section{AXIAL RF COUPLING}

Another novel feature of this design is the use of coaxial coupling through the cathode assembly to a waveguide feed as in figure 7 . This gives strong coupling to the fundamental mode, which is necessary for supplying up to $1 \mathrm{MW}$ of beam power. By adjusting the length of the coaxial region and the position of the shorting plane on the waveguide the coupling factor $(\beta)$, can be varied between 1 and 11 as needed (0-1A of beam 
current). Axial coupling preserves symmetry in the cavity, eliminating any transverse kicks from side coupling ports. DC biased waveguide-coax transitions such as this are presently being used on the SNS [9].

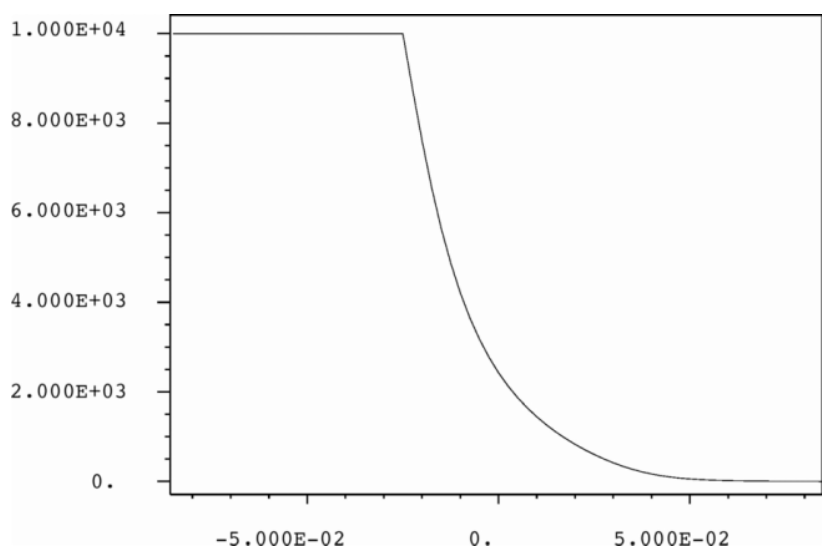

Figure 6. DC Electric potential on axis (V) vs length (m)

RF sources and windows operating at MW power levels are commonplace in L-band and should not be a limiting factor in gun design. HOM damping could also be achieved though this coupler and other dedicated HOM dampers could be added if needed. Frequency tuning can be achieved either by varying the water temperature or changing the position of the cathode. Since moving the cathode also changes the coupling it may be preferable to do this once, correct the coupling with the waveguide stub and subsequently use water temperature for fine-tuning.

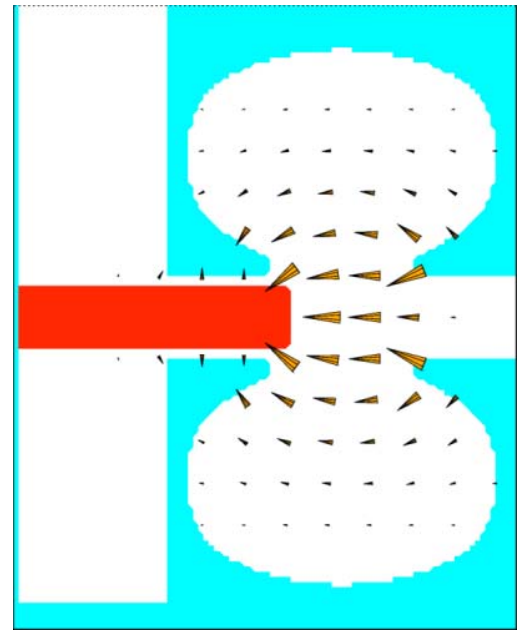

Figure 7 . Re-entrant cavity with waveguide/axial feed.

\section{BEAM DYNAMICS}

Performance of re-entrant RF guns has been shown to be very similar to pillbox guns with the same field strength [10]. If peak electric field on the cathode is in fact the key to controlling space-charge emittance blowup then re-entrant guns should have a significant advantage. Peak fields should also be significantly higher than DC guns. The use of warm technology also allows the use of solenoidal magnetic fields for emittance compensation. Detailed beam dynamics simulations have not yet been done for this gun, however the performance of the similar LUX gun has been extensively studied [11]. Downstream acceleration can be by any appropriate highgradient structures.

\section{CONCLUSIONS}

This re-entrant design shows that peak electric fields on the cathode of up to $20 \mathrm{MV} / \mathrm{m}$ should be achievable in CW mode, with a large safety factor in stress, in a simple structure without resort to exotic materials, cryogenic operation or other complications. The beam dynamics performance of the re-entrant gun is very similar to any other RF gun with comparable fields. The axial power feed avoids any coupler kicks and DC bias on the cathode should enhance quantum efficiency lifetime. This shape optimization is also valid for low rep-rate high peak power guns, which are often limited by surface pulse heating, such as those needed for LCLS or the Tesla XFEL. The re-entrant shape, DC bias scheme for ion clearing and axial coupling for symmetry could also be applied to SRF guns.

\section{REFERENCES}

[1] D. Dowell et. al., "First operation of a Photocathode Radio Frequency Gun Injector at High Duty Factor", Appl, Phys. Lett., Vol. 63, No. 15, 11 October 1993.

[2] A. Zholents, R. Rimmer, O. Walter, W. Wan, M. Zolotorev, "FEL Design for Power Beaming", FreeElectron Laser Challenges II, Proceedings of SPIE, Vol. 3614, p.72, (1999).

[3] R.A. Rimmer et. al., "A High-Gradient High-DutyFactor RF Photo-Cathode Electron Gun”, EPAC 2002, Paris.

[4] J.W. Staples et. al., "The LBNL Femtosource (LUX) $10 \mathrm{kHz}$ Photoinjector", EPAC 2004, Lucerne, Switzerland.

[5] J.W. Staples et. al., "Engineering design of the LUX Photoinjector", EPAC 2004, Lucerne, Switzerland.

[6] "Low temperature mechanical properties of copper and selected copper alloys; a compilation from the literature" ,Richard P. Reed and Ritchie P. Mikesell, [Washington] U.S. Dept.of Commerce, National Bureau of Standards; for sale by the Supt. of Docs., U.S. Govt. Print. Off., 1967, p43.

[7] R.A. Rimmer et. al., "PEP-II RF Cavity Revisited", LBNL CBP Tech Note 197, SLAC LCC-0032 November 1999.

[8] J. Lewellen, "Ion Tracking in Photocathode RF Guns," PRST-AB, Vol 5, 020101 (2002).

[9] M. Stirbet et. al., "RF Conditioning and Testing of Fundamental Power Couplers for SNS Superconducting Cavity Production", these proceedings..

[10] Bill Graves, private communication.

[11] S. Lidia, "Design of injector systems for LUX", EPAC 2004, Lucerne, Switzerland. 